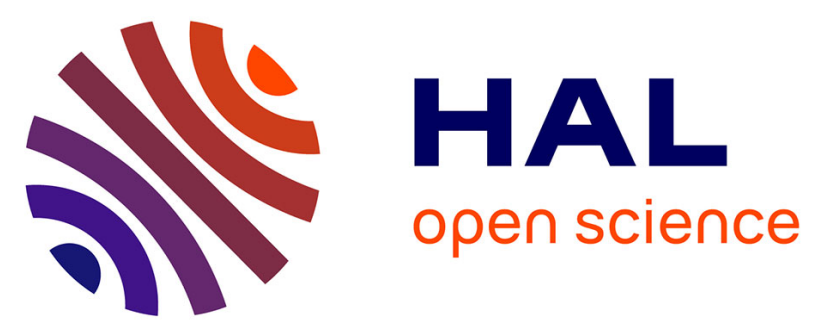

\title{
Probing quantum and classical turbulence analogy in von Kármán liquid helium, nitrogen, and water experiments
}

B Saint-Michel, E Herbert, Julien Salort, Christophe Baudet, Michel Bon-Mardion, Patrick Bonnay, Mickaël Bourgoin, Bernard Castaing, Laurent Chevillard, François Daviaud, et al.

\section{- To cite this version:}

B Saint-Michel, E Herbert, Julien Salort, Christophe Baudet, Michel Bon-Mardion, et al.. Probing quantum and classical turbulence analogy in von Kármán liquid helium, nitrogen, and water experiments. Physics of Fluids, 2014, 26 (12), pp.125109. 10.1063/1.4904378 . hal-01198372

\section{HAL Id: hal-01198372 \\ https://hal.science/hal-01198372}

Submitted on 15 Sep 2015

HAL is a multi-disciplinary open access archive for the deposit and dissemination of scientific research documents, whether they are published or not. The documents may come from teaching and research institutions in France or abroad, or from public or private research centers.
L'archive ouverte pluridisciplinaire HAL, est destinée au dépôt et à la diffusion de documents scientifiques de niveau recherche, publiés ou non, émanant des établissements d'enseignement et de recherche français ou étrangers, des laboratoires publics ou privés. 


\section{AIP | Physics of}

Probing quantum and classical turbulence analogy in von Kármán liquid helium, nitrogen, and water experiments

B. Saint-Michel, E. Herbert, J. Salort, C. Baudet, M. Bon Mardion, P. Bonnay, M. Bourgoin, B.

Castaing, L. Chevillard, F. Daviaud, P. Diribarne, B. Dubrulle, Y. Gagne, M. Gibert, A. Girard, B.

Hébral, Th. Lehner, B. Rousset, and SHREK Collaboration

Citation: Physics of Fluids (1994-present) 26, 125109 (2014); doi: 10.1063/1.4904378

View online: http://dx.doi.org/10.1063/1.4904378

View Table of Contents: http://scitation.aip.org/content/aip/journal/pof2/26/12?ver=pdfcov

Published by the AIP Publishing

\section{Articles you may be interested in}

Superfluid high REynolds von Kármán experiment

Rev. Sci. Instrum. 85, 103908 (2014); 10.1063/1.4897542

High-precision Taylor-Couette experiment to study subcritical transitions and the role of boundary conditions and size effects

Rev. Sci. Instrum. 84, 065106 (2013); 10.1063/1.4807704

Developed quantum turbulence and its decaya)

Phys. Fluids 24, 011301 (2012); 10.1063/1.3678335

Liquid helium inertial jet for comparative study of classical and quantum turbulence Rev. Sci. Instrum. 82, 115109 (2011); 10.1063/1.3661127

The von Kármán Sodium experiment: Turbulent dynamical dynamos Phys. Fluids 21, 035108 (2009); 10.1063/1.3085724






\title{
Probing quantum and classical turbulence analogy in von Kármán liquid helium, nitrogen, and water experiments
}

\author{
B. Saint-Michel, ${ }^{1,2}$ E. Herbert, ${ }^{1,3}$ J. Salort, ${ }^{4}$ C. Baudet, ${ }^{5}$ M. Bon Mardion, ${ }^{6}$ \\ P. Bonnay, ${ }^{6}$ M. Bourgoin, ${ }^{5}$ B. Castaing, ${ }^{4}$ L. Chevillard, ${ }^{4}$ F. Daviaud, ${ }^{1}$ \\ P. Diribarne,${ }^{6}$ B. Dubrulle, ${ }^{1}$ Y. Gagne,${ }^{5}$ M. Gibert,${ }^{7}$ A. Girard, ${ }^{6}$ B. Hébral,${ }^{7}$ \\ Th. Lehner, ${ }^{8}$ and B. Rousset ${ }^{6}$ (SHREK Collaboration) \\ ${ }^{1}$ Laboratoire SPHYNX, CEA/IRAMIS/SPEC, CNRS URA 2464, \\ F-91191 Gif-sur-Yvette, France \\ ${ }^{2}$ Aix Marseille Université, CNRS, Centrale Marseille, IRPHE UMR 7342, \\ 13384 Marseille, France \\ ${ }^{3}$ Université Paris Diderot - Sorbonne Paris Cité, Laboratoire Interdisciplinaire des Énergies \\ de Demain (LIED) - CNRS UMR 8236, Paris, France \\ ${ }^{4}$ Laboratoire de Physique de l'ÉNS de Lyon, CNRS/Université Lyon - F-69364 Lyon \\ CEDEX 7, France \\ ${ }^{5}$ Laboratoire des Écoulements Géophysiques et Industriels, CNRS/UJF/INPG, \\ F-38041 Grenoble CEDEX 9, France \\ ${ }^{6}$ Univ. Grenoble Alpes, INAC-SBT, F-38000 Grenoble, France and CEA, INAC-SBT, \\ F-38000 Grenoble, France \\ ${ }^{7}$ Univ. Grenoble Alpes, Inst NEEL, F-38042 Grenoble, France and CNRS, Inst NEEL, \\ F-38042 Grenoble, France \\ ${ }^{8}$ LUTH, Observatoire Paris-Meudon, 5 Pl. Jules Janssen, F-92195 Meudon CEDEX, France
}

(Received 24 June 2014; accepted 2 December 2014; published online 23 December 2014)

\begin{abstract}
We report measurements of the dissipation in the Superfluid helium high REynold number von Kármán flow experiment for different forcing conditions. Statistically steady flows are reached; they display a hysteretic behavior similar to what has been observed in a 1:4 scale water experiment. Our macroscopical measurements indicate no noticeable difference between classical and superfluid flows, thereby providing evidence of the same dissipation scaling laws in the two phases. A detailed study of the evolution of the hysteresis cycle with the Reynolds number supports the idea that the stability of the steady states of classical turbulence in this closed flow is partly governed by the dissipative scales. It also supports the idea that the normal and the superfluid components at these temperatures $(1.6 \mathrm{~K})$ are locked down to the dissipative length scale. (C) 2014 AIP Publishing LLC. [http://dx.doi.org/10.1063/1.4904378]
\end{abstract}

\section{INTRODUCTION}

At low temperatures, liquid helium 4 undergoes a phase transition, from a classical fluid phase called He I ( $T>2.18 \mathrm{~K}$ at saturated vapor pressure) to a superfluid phase called He II. This superfluid behaves like a two-fluid system, with one normal component, following Navier-Stokes equations, and one superfluid irrotational component, with quantized small scale vortices and zero viscosity (see e.g., Ref. 1). When a superfluid flow is mechanically forced within a pipe, a tank, or past an obstacle, it can become turbulent. ${ }^{2}$ An important open question is how much analogy such quantum turbulence bears with classical turbulence, and how much it can be explained within the above mentioned two-fluid model.

In classical turbulence, at sufficiently large Reynolds number, one distinguishes the (large) "inertial" scales, where viscosity has no influence, and the (small) "dissipative" scales, where viscosity matters. For instance, the energy dissipation is known to be independent of the Reynolds number, thus of the viscosity. It is controlled by the way large scales feed the intermediate inertial ones. In the particular case of isotropic homogeneous turbulence, the energy spectrum exhibits a universal behavior as $k^{-5 / 3}$ when the wave number $k$ belongs to the inertial scales. 
Evidence of the similarity between quantum and classical turbulence trace back to pipe flow experiments ${ }^{3}$ where large-scale pressure drop measurements in the He II phase were found in good agreement with classical turbulence results. Since the pioneering von Kármán liquid helium experiment of Ref. 4, it is also known that the inertial scales energy spectrum does not change when the fraction of normal to superfluid is lowered from $100 \%$ to $20 \%$ and follows the above classical $k^{-5 / 3}$-law. This finding was later confirmed in different geometries and with different forcing conditions in Ref. 5 using both a superfluid jet and grid turbulence. This analogy between normal and quantum turbulence also extends to the energy dissipation, that was found equal in the classical and superfluid phases at large Reynolds numbers. ${ }^{6-11}$ In addition, energy transfers have been found independent of the turbulence nature (quantum or classical) in experiments conducted with Pitot tubes, ${ }^{12}$ down to the smallest experimentally accessible scales, unfortunately much larger than the dissipative scales. ${ }^{13}$

The present experimental state-of-the-art provides strong evidence that quantum turbulence can be described at large scales by classical formulae describing conventional turbulence. A traditional explanation relies on the fact that both components interact with the quantum vortices, resulting in a mutual friction term between the two components in the movement equations. This mutual friction is expected to behave as the difference of the velocity fields of the two components, $v_{\mathrm{n}}$ and $v_{\mathrm{s}}$, with an amplitude proportional to the vortex line density of the superfluid component. Steady superfluid turbulent flows generally minimize this term, locking the normal and superfluid velocity fields together at large scales and inducing a state of coupled turbulence. ${ }^{14,15}$ If this locking is efficient down to below the scale where the viscosity of the normal component damps the normal velocity field gradients, one would have a single velocity field down to this scale. This velocity field would be that of a turbulent fluid with the total density $\rho$ and the viscosity $\mu$ of the normal component, thus with a kinematic viscosity $v_{\text {eff }}=\mu / \rho$. However, the decoupling between the two components could occur at larger scale, and some authors prefer to extract an effective kinematic viscosity from the comparison of the dissipated power and the vortex line density. ${ }^{16}$

In the present paper, we report an even more challenging test of the analogy between quantum and classical turbulence by exploring the response of the system to a forcing asymmetry in the superfluid helium high Reynold number von Kármán flow (SHREK) experiment, a low-temperature forced von Kármán liquid helium experiment providing very high Reynolds measurements in liquid helium both in its classical (above $2.18 \mathrm{~K}$ ) and superfluid phases (below $2.18 \mathrm{~K}$ ). Such response has been previously extensively studied in a smaller version of the experiment involving classical fluids (water or glycerol) ${ }^{17-19}$ and was shown to lead to global bifurcation akin to first or second order phase transition. In the former case, the transition involves both inertial effects and non-trivial Reynolds dependence. ${ }^{19}$ It is therefore a good candidate to probe the effect of a change in the dissipation mechanism, and it will also provide more stringent tests of the two-fluid model and the analogy between quantum and classical turbulence.

\section{GENERALITIES}

\section{A. Experimental setups}

The detailed experimental setup of SHREK is described in Ref. 20. It consists in a cylinder of radius $R=0.39 \mathrm{~m}$ and height $H=1.2 \mathrm{~m}$ (Fig. 1). The fluid is mechanically stirred by a pair of coaxial impellers rotating in opposite directions. The impellers are disks of radius $0.925 R$, fitted with 8 radial aluminum blades of height $0.2 R$ and curvature radius $0.4625 R$. Two senses of rotation can be defined, according to whether the flow is pushed with the convex (+) or concave ( - ) part of the blades. The disks inner surfaces are $1.8 R$ apart setting the axial distance between impellers from blades to blades to $1.4 R$. The impellers rotation rate $f_{1}$ and $f_{2}$ can be varied independently from 0.1 to $2 \mathrm{~Hz}$, delivering a total power ranging from $120 \mathrm{~W}$ at $1.6 \mathrm{~K}$ to $800 \mathrm{~W}$ at $4.5 \mathrm{~K}$.

This experiment benefits from high flexibility of the flow conditions, due to the large variation of helium properties over the available temperature range $(1.6 \mathrm{~K}$ to $5 \mathrm{~K})$. Both superfluid and classical turbulence measurements are possible in the same experiment, with an adjustable fraction of the superfluid component. Therefore, experiments in helium were conducted under a $P=1.1$ bar 


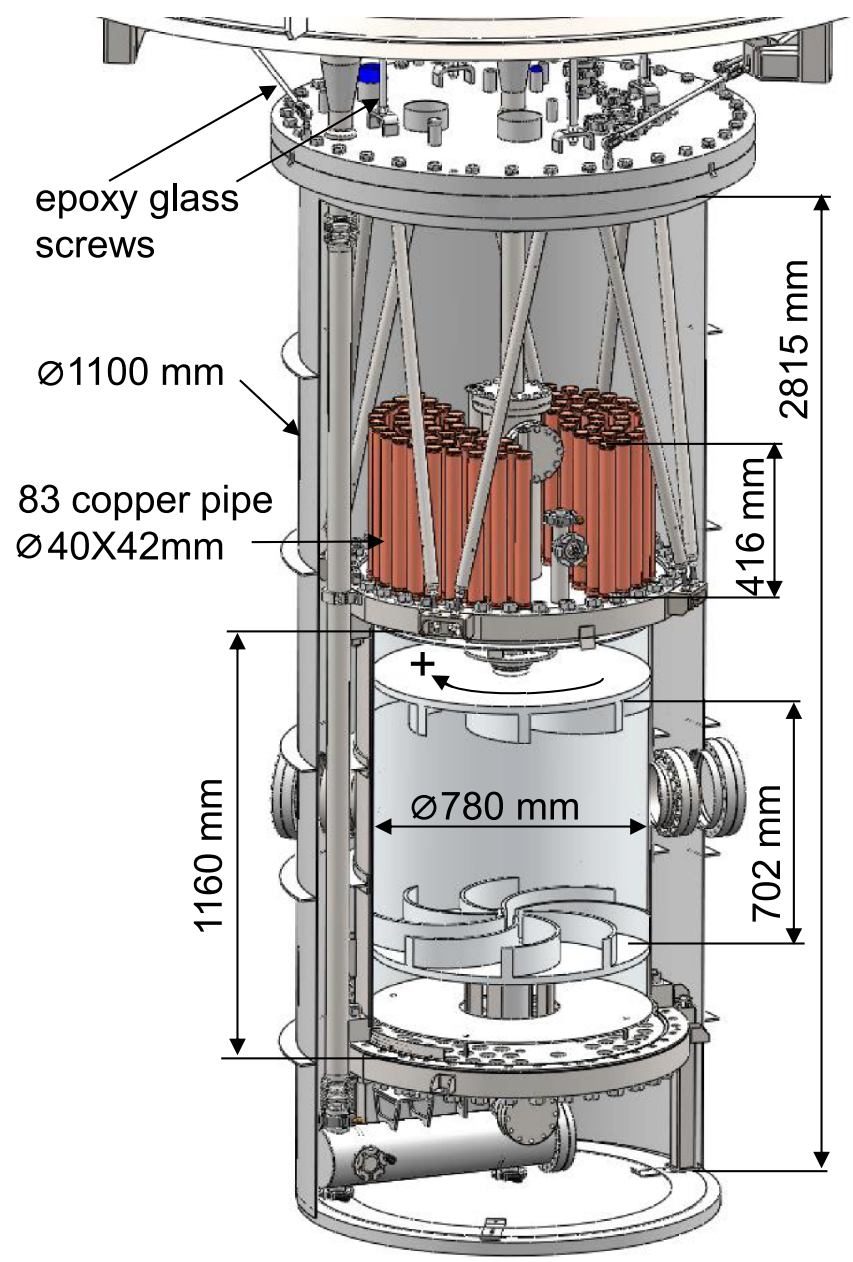

FIG. 1. Schematic view of the SHREK experimental setup and the impellers blade profile. The arrow on the shaft indicates the $(+)$ impeller rotation direction.

pressure at temperatures above $(T=2.62 \mathrm{~K})$ and below $(T=1.63 \mathrm{~K})$ the superfluid transition, to ensure a total fluid density of $\rho=147.3 \mathrm{~kg} / \mathrm{m}^{3}$ in each case. In the He II regime, the superfluid fraction was about $85 \%$. Additional classical turbulence experiments have also been performed using nitrogen at $284 \mathrm{~K}$ and 4 bar.

Torque measurements at each impeller are performed with SCAIME technology and provide values over the $\mathrm{kHz}$ range of $C_{1}$ and $C_{2}$, being, respectively, the torque applied to the bottom and top shafts. Following the procedure described in Ref. 21, they are calibrated using measurements at different mean frequencies, so as to remove spurious contributions from genuine offsets or mechanical friction.

The Saclay experiment is a 1:4 scale version of the SHREK experiment using water and water-glycerol mixtures as primary fluids. The geometry of the water vessel is identical to SHREK, using steel impellers with 16 blades for Reynolds numbers between 50 and 50000 and polycarbonate impellers with 8 blades otherwise. ${ }^{17,21,22}$ The non-dimensional curvature radius and height of the blades are identical to those of SHREK; the polycarbonate impellers are thus an exact 1:4 version of the SHREK impellers. The global mechanical set-up is however much simpler since no specific cooling or thermal isolation system is required. Torque measurements are provided by the same model of SCAIME torquemeters, which have been calibrated using the same procedure. In addition, the three components of the instantaneous velocity field are measured in a vertical middle plane using a stereoscopic PIV (Particle Image Velocimetry) equipment provided by DANTEC Dynamics. 


\section{B. Control parameters and diagnostics}

The control parameters of the studied von Kármán flows are the Reynolds number $\mathrm{Re}$ $=\pi\left(f_{1}+f_{2}\right) R^{2} / v$, where $v=\mu_{n} / \rho$ is the kinematic viscosity of the normal fluid component, as discussed in the Introduction, which controls the intensity of turbulence, and the rotation number $\theta=\left(f_{1}-f_{2}\right) /\left(f_{1}+f_{2}\right)$, which controls the forcing asymmetry. Choosing $R$ and $\Omega^{-1}=\left(\pi\left(f_{1}\right.\right.$ $\left.\left.+f_{2}\right)\right)^{-1}$ as unit of length and time, we compute the non-dimensional value $K_{p 1}$ and $K_{p 2}$ of the torques as $K_{p i}=C_{i} /\left(\rho R^{5} \Omega^{2}\right)$, where $\rho$ is the density of the working fluid. We compute two diagnostics: the mean non-dimensional torque $K_{p}=\left(K_{p_{1}}+K_{p_{2}}\right) / 2$ and the response to asymmetry $\Delta K_{p}=\left(K_{p_{1}}-K_{p_{2}}\right)$. In the exact counter-rotating case $(\theta=0)$, the non-dimensional mean dissipation is $\left(C_{1}+C_{2}\right) \Omega /\left(\rho R^{5} \Omega^{3}\right)=2 K_{p}$. We present results obtained from the two experiments and four main types of fluids, under operating conditions and characteristics that are summarized in Table I. Hence, including water-glycerol mixtures, these results cover Reynolds numbers in the $\left[5 \times 10^{2} ; 10^{8}\right]$ range, rotation numbers between -1 and 1 for the $(-)$ sense of rotation, and $\operatorname{Re} \in\left[5 \times 10^{2} ; 10^{8}\right]$ at $\theta=0$ for the (+) sense.

\section{Flow geometry}

In the water experiment, at any Reynolds number and for any sense of impeller rotation, a symmetric mean flow, as depicted in Figure 2, can be statistically reached for exact counter-rotation $(\theta=0) .{ }^{17}$ This mean flow consists in two counter-rotating toroidal cells separated by a shear layer; it is modulated by a poloidal flow induced by Ekman pumping from the impellers. For a given Reynolds number, the introduction of a forcing asymmetry through an increase of the control parameter $|\theta|$ induces a breakdown of the mean flow symmetry with respect to the equatorial axis - called $\mathcal{R}_{\pi}$ symmetry - (see Fig. 2, bottom panels), towards a non-symmetric steady state.

Since our impellers have curved blades, the sensitivity to forcing asymmetry and the nature of the transition from the two-cell state to the one-cell state depends on the forcing direction; for the (+) direction, the change is continuous and occurs via a sequence of increasingly non-symmetric two-cell states, with one cell becoming larger at the expense of the other; in the (-) direction, the change is discontinuous for large enough Reynolds numbers $(\operatorname{Re} \geq 4000)$ and becomes hysteretic for higher Reynolds numbers $(\operatorname{Re} \geq 10000):{ }^{17,22,23}$ starting both impellers at the same time for $\theta$ very close to 0 , the system reaches two-cell "symmetric" states, which verify almost exactly $K_{p 1}=K_{p 2}$. A slight variation of $\theta$ on this branch triggers a global bifurcation with a dramatic increase of the mean torque. These new states display only one recirculation cell, one impeller pumping the fluid from the whole vessel. They aggregate on two branches called $\left(b_{1}\right)$ (mainly for $\theta>0$ ) and $\left(b_{2}\right)$ (mainly for $\theta \leq 0$ ), which are images of each other through $\mathcal{R}_{\pi}$ symmetry. Once on $\left(b_{1}\right)$ and $\left(b_{2}\right)$ branches, it is never possible to reach again the $(s)$ state, which is thus only observed while starting our impellers at the exact same time. In addition, the $\left(b_{1}\right)$ and $\left(b_{2}\right)$ branches are hysteretic, $\left(b_{1}\right)$ (resp. $\left(b_{2}\right)$ ) being stable for $\theta<-\theta_{*}$ (resp. $\theta>\theta_{*}$ ), where $\theta_{*} \geq 0$ depends on the Reynolds number.

TABLE I. Summary of the four fluid configurations studied in the present paper and their main properties (density $\rho$, viscosity $v$, Reynolds number Re) under operating conditions (pressure $P$, temperature $T$, mean impeller frequency $F=\left(f_{1}+f_{2}\right) / 2$, and cylinder radius $R$ ). In the He II superfluid regime, the effective viscosity $v_{\text {eff }}$ is reported.

\begin{tabular}{llcccccc}
\hline \hline Fluid & $P$ & $T$ & $\Omega$ & $R$ & $\rho$ & $v$ \\
$(\mathrm{bar})$ & $(\mathrm{K})$ & $(\mathrm{Hz})$ & $(\mathrm{m})$ & $\begin{array}{c}\rho \\
\left(\mathrm{kg} / \mathrm{m}^{3}\right)\end{array}$ & $\left(\mathrm{m}^{2} / \mathrm{s}\right)$ & $\mathrm{Re}$ \\
\hline $\mathrm{He} \mathrm{I}$ & 1.1 & 2.62 & $0.1-0.6$ & 0.4 & 147.3 & $2 \times 10^{-8}$ & $5 \times 10^{6}-3 \times 10^{7}$ \\
$\mathrm{He}$ II & 1.1 & 1.63 & $0.1-0.6$ & 0.4 & 147.3 & $1 \times 10^{-8}$ & $1 \times 10^{7}-6 \times 10^{7}$ \\
$\mathrm{~N}_{2}$ & 3.73 & 284 & 1.8 & 0.4 & 4.4 & $4 \times 10^{-6}$ & $5 \times 10^{5}$ \\
$\mathrm{H}_{2} \mathrm{O}$ & 1.8 & 300 & $2-15$ & 0.1 & 1000 & $1 \times 10^{-6}$ & $1 \times 10^{5}-1 \times 10^{6}$ \\
\hline \hline
\end{tabular}



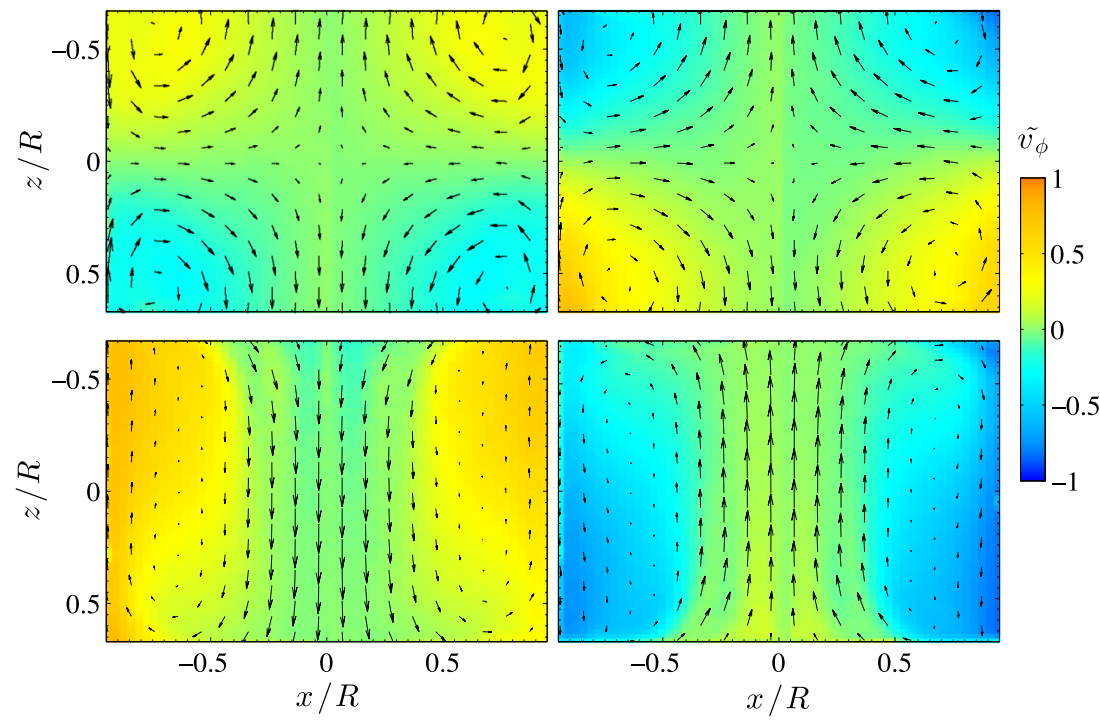

FIG. 2. Mean turbulent velocity fields observed using particle image velocimetry in the 1:4 Saclay water experiment ${ }^{19,21}$ for $\theta=0$. False colors, non-dimensional azimuthal velocity $\tilde{v_{\phi}}=v_{\phi} / 2 \pi R \Omega$ (same for all panels). Arrows, radial and vertical velocities. From left to right and top to bottom: symmetrical two-cell state $(s)$ in the $(+)$ sense, symmetrical two-cell state $(s)$ in the $(-)$ sense, one-cell $\left(b_{1}\right)$ state, and one-cell $\left(b_{2}\right)$ state. The arrow scale has been reduced in the bottom two panels by a factor 4 to improve readability.

\section{RESULTS}

\section{A. Dissipation}

In this section, we study the impact of the nature of turbulence on the hysteresis cycle. As mentioned above, there is now overwhelming evidence that in classical homogeneous and isotropic turbulent flows of typical fluctuating velocity $u$ and typical length $L$, the mean energy dissipation rate does not vanish in the limit of vanishing viscosity, but instead converges to a finite universal limit when properly scaled by $u^{3} / L .^{24,25}$ This mass dissipation rate can be related to the Kolmogorov constant. ${ }^{26,27}$ There is presently no rigorous derivation of this property from the Navier-Stokes equations, so we cannot rule out a priori a dependence of this limit on the nature of energy dissipation. For a non homogeneous and non isotropic flow such as the von Kármán flow, the non-dimensional mean energy dissipation is given by $2 K_{p}$; a crucial question is therefore whether $K_{p}$ undergoes a variation when the superfluid fraction increases.

Measurements of the non-dimensional dissipation $K_{p}$ for classical fluids have been done in the Saclay 1:4 experiment for $\theta=0$ and Reynolds numbers between 50 and $10^{6}$; they are summarized in Fig. 3. The dissipation $K_{p}$ saturates at $\mathrm{Re} \sim 10^{5}$ in both $(+)$ and (-) rotation senses. ${ }^{23}$ SHREK experiments extend these measurements up to $\operatorname{Re}=6 \times 10^{7}$ and confirm this saturation without any ambiguity in the three steady regimes: the two-cell symmetric states in $(+)$ and $(-)$ senses and the one-cell state in the (-) sense (see Fig. 3). The overlap of measurements in $\mathrm{N}_{2}$ (SHREK) and in $\mathrm{H}_{2} \mathrm{O}$ (Saclay), performed at similar Reynolds numbers, but in different experiments, confirms the reliability of our calibrations process and indicates that no additional bearing due to mechanical friction can explain this saturation. We have also measured the dissipation in the superfluid phase (symbols with black outline), in the (-) rotation sense. The result is also shown in Fig. 3 using the effective Reynolds number. One sees that the dissipation is equal in the classical and in the superfluid phases, thereby confirming an earlier result obtained in a von Kármán flow driven by impellers with straight blades. ${ }^{6}$

\section{B. Torque asymmetry response}

We have further investigated the universality of the energy dissipation when the flow symmetry is broken, imparting a differential rotation of the impellers, or in other terms imposing $\theta \neq 0$. In this 




FIG. 3. Variations of $K_{p}=\left(K_{p 1}+K_{p 2}\right) / 2$ as a function of Re at $\theta=0$ for various fluids. Measurements for Reynolds numbers up to $5 \times 10^{4}$, obtained from the Saclay experiment, are taken from Ref. 23 using 16 blade impellers (see Sec. II A). Pink upwards pointing triangles, one-cell $\left(b_{1}\right)(-)$ regime in Saclay experiment; pink downwards pointing triangles, same regime in SHREK (nitrogen); pink right pointing triangles, same regime in helium. Orange diamonds, two-cell $(s)(-)$ regime of Saclay experiments. Orange squares, same regime in helium, in SHREK. Green circles and stars, two-cell (+) regimes, respectively, for Saclay and SHREK nitrogen experiments. Green squares, SHREK helium experiments. Symbols with black outline account for superfluid experiments. Dotted lines are eye-guides. The black dashed line accounts for viscous stirring, $K_{p} \propto \operatorname{Re}^{-1}$.

case, the torques measured at the two impellers become different. It is therefore useful to study their individual behavior. This is done in the classical regime in Fig. 4-left, for the experiment operating in the (-) sense and for the 3 different fluids, at different Reynolds numbers. All the curves collapse approximately on two master curves, one for each impeller, corresponding to the water experiments of Saclay. For large enough $\theta$, the two curves are single valued, the largest non-dimensional dissipation being observed for the impeller rotating at the largest frequency. In the range $|\theta| \leq 0.4$, the dissipation becomes double-valued, following the multi-stability of large-scale flow solutions. In this region, the collapse quality is lower; the $\mathrm{H}_{2} \mathrm{O}$ and $\mathrm{N}_{2}$ fluids are multi-valued on a smaller interval than $\mathrm{He}$ I. This difference will be further discussed later in this section.

Overall, the collapse observed in Fig. 4 confirms that the non-dimensional dissipation in the classical regime is independent of $\operatorname{Re}$ above $\operatorname{Re} \geq 10^{5}$, at any $\theta$. For comparison, we provide in Fig. 4-right, the torque measurements at various $\theta$ 's in the regime with $85 \%$ superfluid. One sees that they collapse on the same two master curves. In this context, while it is tempting to call for singularities to explain anomalous dissipation in homogeneous and isotropic turbulence, as initially

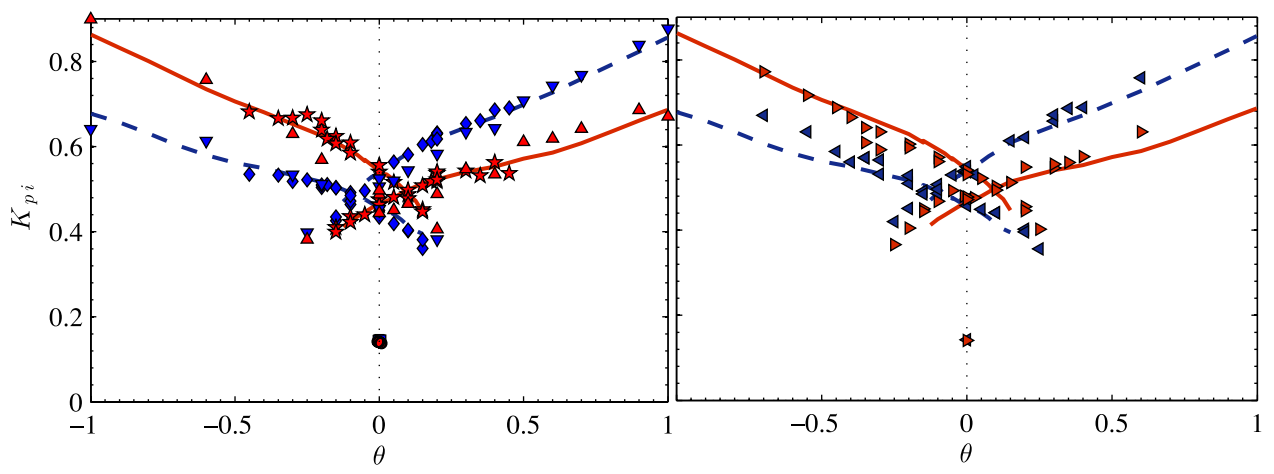

FIG. 4. Non-dimensional torques $K_{p}$ corrected with $\theta=0$ value to ensure $\theta \rightarrow-\theta$ symmetry, varying with $\theta$ for various fluids, corresponding to different Reynolds number ranges (see Table I). Left: blue diamonds and red stars are, respectively, the lower and upper torques in nitrogen experiments. Blue downwards pointing triangles and red upwards pointing triangles, same quantities in He I. Right: blue left pointing triangles and red right pointing triangles, same quantities in He II. For both graphs, the red solid line and the blue dashed line recall the values of $K_{p}(\theta)$ for Saclay experiments in water. 


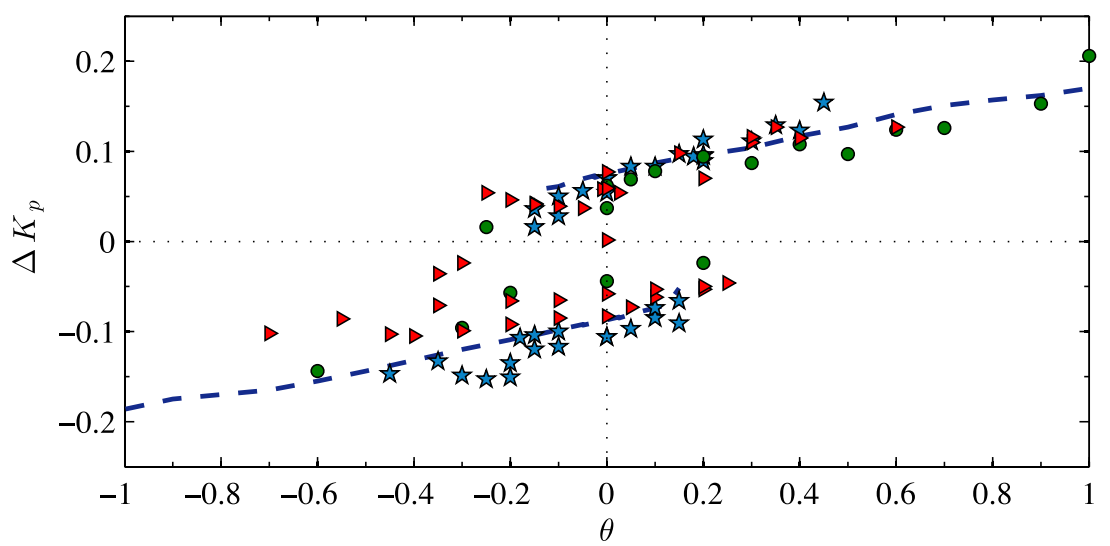

FIG. 5. Shifted values of $\Delta K_{p}(\theta)$ in SHREK to ensure symmetry with respect to $\theta=0$ (same process as in Figure 4). Teal stars, nitrogen experiment; green circles, liquid helium; red right pointing triangles, superfluid helium. Blue dashed line recalls the $\Delta K_{p}$ values of the Saclay water experiments.

suggested by Onsager, ${ }^{28-30}$ it appears that the large-scale mean flow is instead of crucial importance to determine the limiting value of $K_{p}$ in a von Kármán flow. We can also infer that the very nature of the dissipation process does not itself select the large scale flow, justifying a posteriori the statistical physics descriptions of the von Kármán steady states. ${ }^{18,31}$

A convenient visualization of the hysteresis cycle relies on the normalized torque difference $\Delta K_{p}$, plotted as a function of $\theta$ (see Figure 5); for the Saclay experiments (dashed line), the two branches $\left(b_{1}\right)$ and $\left(b_{2}\right)$ are clearly visible, the upper $\left(b_{1}\right)$ branch, starting from $-\theta^{*}$ to $\theta=1$, and the lower branch $\left(b_{2}\right)$, starting from $\theta=-1$ to $\theta^{*}$, drawing a cycle reminiscent of ferromagnetic systems. SHREK data are more scattered, especially compared to the individual torques, but they roughly collapse around the two branches of the classical hysteresis cycle.

Eventually, we have investigated the influence of the Reynolds number and the turbulent nature on the fine properties of our hysteresis cycles. The shape of these cycles in the $\left(\theta, \Delta K_{p}\right)$ plane suggests the definition of two relevant cycle quantities, which are the cycle width $2 \theta^{*}$ and the cycle height $\Delta K_{0}$, defined as the difference of $\Delta K_{p}$ between the $\left(b_{1}\right)$ and $\left(b_{2}\right)$ branches for a symmetrical forcing $(\theta=0)$. These quantities have been plotted in Figure 6 for all experimental conditions. For Reynolds numbers around $\operatorname{Re} \sim 5 \times 10^{5}$ (water and nitrogen experiments), the cycle width is roughly $2 \theta^{*}=0.3 \pm 0.03$, while at $\operatorname{Re} \sim 2 \times 10^{7}$ (He I fluid), it reaches $2 \theta^{*}=0.45 \pm 0.03$. For the largest values of the Reynolds number, the cycle height $\Delta K_{0}$ is actually slightly smaller than at lower Reynolds numbers. This trend is confirmed in the superfluid case (He II), with a hysteresis width also of the order of again $2 \theta^{*}=0.5 \pm 0.03$ and height of $\Delta K_{0}=0.13 \pm 0.03$.

While the high dispersion of the results of $K_{p}(\theta)$ limits the precision on $\Delta K_{0}$ in SHREK (see Figure 5), the precision of the value of $\theta^{*}( \pm 0.03)$ remains fair compared to the water experiments (where the precision on $\theta^{*}$ is 0.01 ) because it is linked to an imposed, well controlled quantity, namely, the rotation frequency of the disks. Overall, one observes a well-defined trend as a function of the Reynolds number confirming the previous results obtained only in water experiments. ${ }^{19}$ As far as classical fluids are concerned, the present experiment demonstrates that the width of the hysteresis cycle increases with Reynolds numbers leading to the following picture at infinite Reynolds numbers: after reaching a one-cell state, the flow remains stuck in it. It also shows that the origin of the transition from one branch to the other when $\theta$ is increased cannot be attributed to the sole inertial scales, as it depends on the viscosity for Reynolds numbers well above the transition to turbulence. Moreover, the values observed in the superfluid experiment and plotted using the effective Reynolds number fit fairly well with the classical fluid experiments.

While the macroscopic similarity between quantum and classical turbulence is clearly established (pressure drop, ${ }^{3,32}$ drag crisis, ${ }^{9}$ etc.), the extension of this analogy down to smaller inertial scales, though now widely accepted, is mainly based on one-point velocity measurements. ${ }^{4,6,12}$ However, this does not formally prove that the underlying physical mechanisms are identical above 


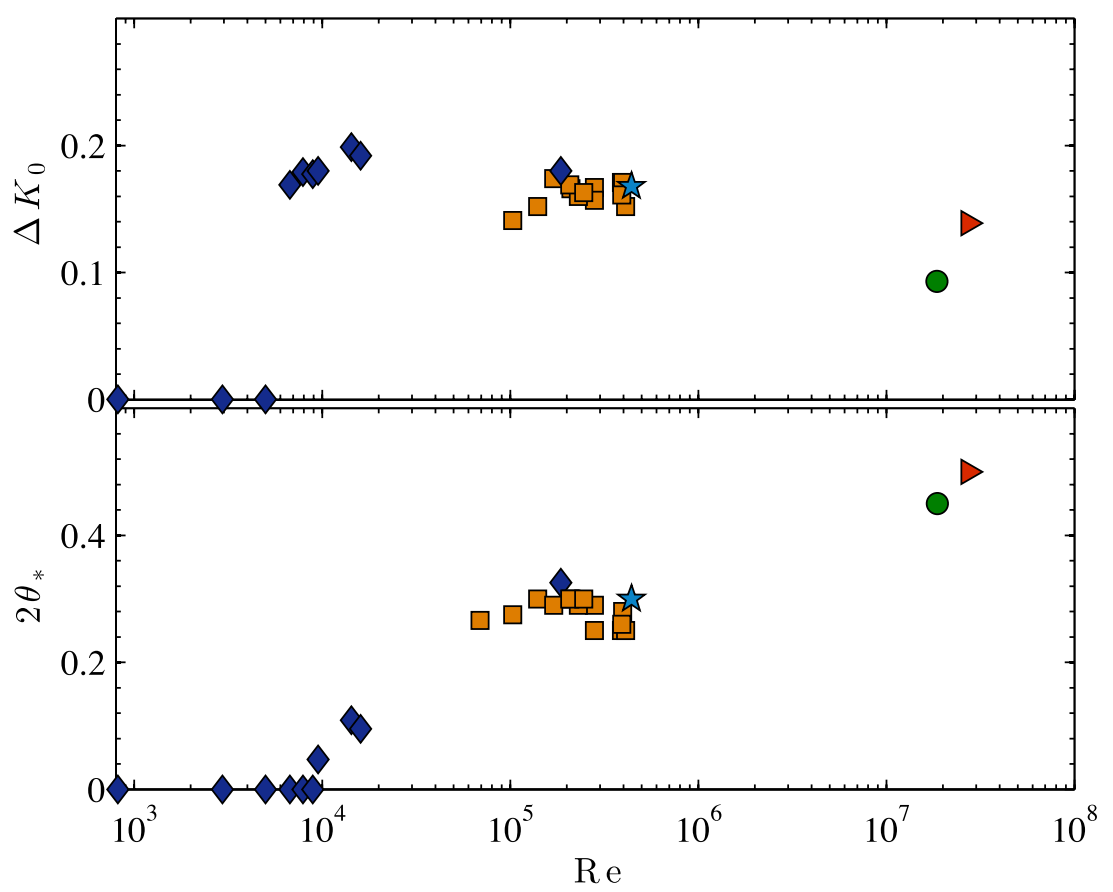

FIG. 6. Top: height $\Delta K_{0}$ of the hysteresis loop plotted in Fig. 5 as a function of the Reynolds number. Bottom: width $2 \theta^{*}$ of the hysteresis cycle. Blue diamonds (resp. orange squares), Saclay experiment in water with 16 blade (8 blade) impellers; teal stars, SHREK experiment in nitrogen; Green circles, in liquid helium; red right pointing triangles, in superfluid helium.

and below the superfluid transition. For example, some classical flows do not follow Kolmogorov dissipation scalings and yet lead to Kolmogorov-like one-point velocity statistics, like $-5 / 3$ spectra. ${ }^{33}$ For this reason, the strengthening of the analogy between the two types of turbulence, down to the small scales, requires to go beyond local velocity measurements, and one example is the analysis of the asymmetry in the SHREK experiment.

\section{DISCUSSION}

Exploring the properties of a global bifurcation in a liquid helium experiment, we have been able to probe the analogy between forced classical and superfluid turbulence in a von Kármán geometry. Our first result is the confirmation, for a vast range of forcing conditions, that the injected energy in the flow is dissipated in the same way in the purely viscous flow (i.e., $T>2.18 \mathrm{~K}$ ) and in the two-component fluid (i.e., $T<2.18 \mathrm{~K}$ ). This was expected since the energy transfers are controlled by the large scales, where the two components of the He II phase are locked by the mutual friction. This also confirms that the very nature of the microscopic dissipation mechanism does not itself select the large scale flow in von Kármán experiments, as it has been proposed in a statistical physics point of view. ${ }^{18}$

Let us discuss now what our results imply about the flipping process from one branch of the hysteresis cycle to the other. This process involves some range of scales in the flow, from the large scale $R$ to some small scale $a_{0}$. This small scale could be the equivalent of the correlation length in the magnetic analogy developed in Ref. 18. It could also be the size of the boundary layers along the blades. The comparative experiments conducted in water (in the 1:4 experiment) and in $\mathrm{N}_{2}$ at the same Reynolds numbers confirm that this distance $a_{0}$ does not depend on tiny details of the experiment, such as the surface roughness of the blades.

However, the continued dependence of $\theta^{*}$ with the Reynolds number, in particular for flows well above the transition to turbulence $\left(\operatorname{Re} \geq 10^{4}\right)$, indicates that $a_{0}$ is slightly larger or of the same order as the dissipative length $\eta$, which is estimated to be of order $\eta \simeq 7.0 \times 10^{-7} \mathrm{~m}$ for $K_{p}=0.5$ 
and $\operatorname{Re}=5 \times 10^{7}$. Even for Reynolds numbers around $\mathrm{Re} \simeq 10^{6}$, this small scale $a_{0}$ will not exceed $1.2 \times 10^{-5} \mathrm{~m}$, smaller than the resolution of available local probes of the velocity field, as hot wires or laser Doppler velocimetry. This high-Reynolds dependence contrasts with the more common pressure drop and drag crisis experiments which are governed by the larger, integral scales, where asymptotic regimes are achieved above the transition to turbulence for classical fluids. Additionally, we observe that the width of the hysteresis cycle $\theta^{*}$ is the same for both phases for the same disks velocity. The width of the hysteresis cycle $\theta^{*}$ being sensitive to scales down to $a_{0}$, the similarity we observe thus suggests that the normal and superfluid components are locked down to (at least) $a_{0}$.

It is finally possible to obtain a rough estimate of the typical inter-vortex distance $\delta$ in our flow. It can be deduced from the considerations of Ref. 34, (Eq. 4) as was done in Ref. 20, knowing the non-dimensional dissipation $K_{p}$. The inter-vortex distance is estimated to be $\delta \approx 1.0 \times 10^{-6} \mathrm{~m}$. This distance is therefore of the same order as the Kolmogorov dissipative scale $\eta$.

In conclusion, our results confirm the components locking down to a scale $a_{0}$ much smaller than the ones accessible through available local probes. The scale $a_{0}$ is slightly larger (or of the same order) than $\eta$, the classical Kolmogorov dissipative scale, and decreases with increasing Reynolds number. These results are in agreement with some models suggesting that the superfluid behaves as a classical fluid of the same density and dynamical viscosity as the normal component. ${ }^{12}$

\section{ACKNOWLEDGMENTS}

We thank the CEA, the CNRS, and contract ANR-09-BLAN-0094-01 for support, F. Bancel, L. Monteiro, Ph. Charvin, C. Gasquet, and V. Padilla for technical support, and Ph.-E. Roche for experimental design support. This work is also supported by the European Community Framework Programme 7, EuHIT-European High-performance Infrastructures in Turbulence, Grant Agreement No. 312778.

${ }^{1}$ L. Skrbek and K. R. Sreenivasan, "Developed quantum turbulence and its decay,” Phys. Fluids 24, 011301 (2012).

${ }^{2}$ L. Skrbek, "Quantum turbulence,” J. Phys.: Conf. Ser. 318, 012004 (2011).

${ }^{3}$ P. L. Walstrom et al., "Turbulent flow pressure drop in various He II transfer system components," Cryogenics 28 101-109 (1988).

4 J. Maurer and P. Tabeling, "Local investigation of superfluid turbulence," EPL 43, 29 (1998).

5 J. Salort et al., "Turbulent velocity spectra in superfluid flows," Phys. Fluids 22, 125102 (2010); D. Duri et al., "Liquid helium inertial jet for comparative study of classical and quantum turbulence,” Rev. Sci. Instrum. 82, 115109 (2011).

${ }^{6} \mathrm{M}$. Abid et al., "Experimental and numerical investigations of low-temperature superfluid turbulence," Eur. J. Mech. B-Fluid 17, 665 (1998)

${ }^{7}$ M. Blazkova et al., "Transition from laminar to turbulent drag in flow due to a vibrating quartz fork," Phys. Rev. E 75 , 025302(R) (2007).

${ }^{8}$ D. I. Bradley et al., "Transition to turbulence for a quartz tuning fork in superfluid 4He," J. Low Temp. Phys 156, 116 (2009).

${ }^{9}$ M. R. Smith et al., "Observed drag crisis on a sphere in flowing He I and He II," Phys. Fluids 11, 751 (1999).

${ }^{10}$ D. I. Bradley et al., "Turbulent drag on a low-frequency vibrating grid in superfluid $4 \mathrm{He}$ at very low temperatures," Phys. Rev. B 85, 224533 (2012).

${ }^{11}$ T. Zhang and S. W. Van Sciver, "Large-scale turbulent flow around a cylinder in counterflow superfluid 4He," Nat. Phys. 1, 36 (2005)

12 J. Salort et al., "Energy cascade and the four-fifths law in superfluid turbulence," EPL 97, 34006 (2012).

${ }^{13}$ D. Duri, "Mise en évidence expérimentale de l'intermittence dans un jet cryogénique turbulent d'hélium normal et superfluide," Ph.D. thesis, Université Joseph Fourier, 2012.

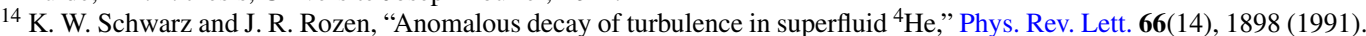

${ }^{15}$ C. F. Barenghi, C. L. Swanson, and R. J. Donnelly, "Emerging issues in helium turbulence," J. Low. Temp. Phys. 100(5-6), 385 (1995)

${ }^{16}$ W. F. Vinen and J. J. Niemela, “Quantum turbulence,” J. Low. Temp. Phys. 128(5-6), 167 (2002).

${ }^{17}$ F. Ravelet $e t$ al., "Multistability and memory effect in a highly turbulent flow: Experimental evidence for a global bifurcation," Phys. Rev. Lett. 93, 164501 (2004).

${ }^{18}$ P.-P. Cortet et al., "Experimental evidence of a phase transition in a closed turbulent flow," Phys. Rev. Lett. 105, 214501 (2010); "Susceptibility divergence, phase transition and multistability of a highly turbulent closed flow," J. Stat. Mech. 2011 P07012 (2011).

${ }^{19}$ B. Saint-Michel, "L'écoulement de von Kármán comme paradigme de la physique statistique hors de l'équilibre," Ph.D thesis, Université Pierre et Marie Curie, 2013.

${ }^{20}$ B. Rousset et al., "Superfluid High REynolds von Kámán experiment,” Rev. Sci. Instrum. 85, 103908 (2014).

${ }^{21}$ L. Marié, "Transport de moment cinétique et de champ magnétique par un écoulement tourbillonnaire turbulent: Influence de la rotation," Ph.D. thesis, Université Paris Diderot, 2003.

${ }^{22}$ B. Saint-Michel, B. Dubrulle, L. Marié, F. Ravelet, and F. Daviaud, "Influence of Reynolds number and forcing type in a turbulent von Kármán flow,” New J. Phys. 16, 063037 (2014). 
${ }^{23}$ F. Ravelet et al., "Supercritical transition to turbulence in an inertially driven von Kármán closed flow," J. Fluid. Mech. 601, 339 (2008).

${ }^{24}$ U. Frisch, Turbulence (Cambridge University Press, 1995).

${ }^{25}$ K. R. Sreenivasan, "An update on the energy dissipation rate in isotropic turbulence," Phys. Fluids 10, 528529 (1998)

${ }^{26}$ S. B. Pope, Turbulent flows (CUP, Cambridge, 2000).

${ }^{27}$ L. Chevillard et al., "A phenomenological theory of Eulerian and Lagrangian velocity fluctuations in turbulent flows," C.R. Physique 13, 899 (2012).

${ }^{28}$ L. Onsager, "Statistical hydrodynamics," Nuovo Cimento 6(Suppl.), 279287 (1949).

${ }^{29}$ J. Duchon and R. Robert, "Inertial energy dissipation for weak solutions of incompressible Euler and Navier-Stokes equations," Nonlinearity 13, 249255 (2000).

${ }^{30}$ G. L. Eyink and K. R. Sreenivasan, "Onsager and the theory of hydrodynamic turbulence,” Rev. Mod. Phys. 78, 87135 (2006).

${ }^{31}$ R. Monchaux et al., "Fluctuation-dissipation relations and statistical temperatures in a turbulent von Kármán flow," Phys. Rev. Lett. 101(17), 174502 (2008).

32 B. Rousset et al., "Pressure drop and transient heat transport in forced flow single phase helium II at high Reynolds numbers," Cryogenics 34, 317-320 (1994).

${ }^{33}$ J. Nedić et al., "Axisymmetric turbulent wakes with new nonequilibrium similarity scalings," Phys. Rev. Lett. 111, 144503 (2013).

${ }^{34}$ S. Babuin et al., "Effective viscosity in quantum turbulence: A steady-state approach,” EPL 106, 24006 (2014) 